\title{
A PREOCUPAÇÃO COM O MEIO AMBIENTE E O CONSUMO: UM ESTUDO COM PROFESSORES DE ADMINISTRAÇÃO DO BRASIL
}

\author{
Márcia Zampieri Grohmann* \\ Daiane Lindner Radons** \\ Aline Nadalin Velter*** \\ Luciana Flores Battistella****
}

RESUMO: A preocupação da sociedade em relação ao meio ambiente cresce e afeta o comportamento dos consumidores. O artigo utiliza a Teoria do Comportamento Planejado (AJZEN, 1991) para compreender a importância do meioambiente de processo de compra. Com uma pesquisa quantitativa e descritiva que utilizou os modelos de Enoki et al. (2008) e Veiga et al. (2006), realizada com 454 professores de cursos de administração em todo o Brasil, identificou-se que: os respondentes concordam que o consumo de produtos ecologicamente corretos traria benefícios ao meio ambiente $(\mu=4,55)$ e possuem intenção moderadamente alta de adquirir tais produtos $(\mu=3,74)$; as normas subjetivas são antecedentes da intenção de compra $\left(\mathrm{R}^{2}=16,5 \%\right)$; as atitudes são antecedentes da intenção de compra de produtos pró-ambientais $\left(\mathrm{R}^{2}=9,3 \%\right)$ e que a intenção de compra tem forte impacto $\left(\mathrm{R}^{2}=54,3 \%\right)$ no comportamento de compra de produtos pró-ambientais. Portanto, conclui-se que os professores pesquisados já desenvolveram preocupações com $o$ meio ambiente e que isto afeta em seus comportamentos de compra.

PALAVRAS-CHAVE: Consumo; Meio Ambiente; Teoria do Comportamento Planejado.

\section{CONCERN ON THE ENVIRONMENT AND CONSUMPTION: A STUDY WITH PROFESSORS OF ADMINISTRATION IN BRAZIL}

ABSTRACT: Society's concern on the environment is continually on the increase and affects consumers' behavior. Current study employs the Theory of Planned Behavior (Ajzen, 1991) to describe the importance of the environment with the

\footnotetext{
Doutora em Engenharia de Produção pela UFSC; Docente Associada do Departamento de Ciências Administrativas e do Programa de Pós-Graduação em Administração da Universidade Federal de Santa Maria (UFSM), Santa Catarina, Brasil; E-mail de correspondência: marciazg@gmail.com marciazg@gmail.com

** Mestre em Administração pela Universidade Federal de Santa Maria (UFSM); Assistente em Administração da Universidade Federal da Fronteira Sul (UFFS), Santa Catarina, Brasil.

*** Mestre em Administração pela Universidade Federal de Santa Maria (UFSM), Rio Grande do Sul, Brasil.

**** Doutora em Engenharia de Produção pela UFSC; Docente Associada do Departamento de Ciências Administrativas e do Programa de Pós-Graduação em Administração da Universidade Federal de Santa Maria (UFSM), Santa Catarina, Brasil.
} 
purchase process. Current quantitative and descriptive research employed models by Enoki et al. (2008) and Veiga et al. (2006) with 454 professors of Administration Courses in Brazil and asked whether the interviewed agreed that the consumption of ecologically correct products are beneficent to the environment $(\mu=4.55)$; whether they have a moderate desire to purchase the products $(\mu=3.74)$; whether subjective norms antecede purchase intention $\left(\mathrm{R}^{2}=16.5 \%\right)$; whether attitudes antecede the purchase intention for buying pro-environment products $\left(\mathrm{R}^{2}=9.3 \%\right)$ and whether purchase intention has a strong impact $\left(\mathrm{R}^{2}=54.3 \%\right)$ on the purchase behavior of pro-environment products. Results show that the interviewed professors were already concerned with the environment and affected their purchasing behavior.

KEY WORDS: Consumption; Environment; Theory of Planned Behavior.

\section{INTRODUÇÃO}

Impulsionada por uma crise ecológica, a preocupação da sociedade em relação ao meio ambiente cresce no decorrer dos anos. Segundo Tsarenko et al (2013), por meio de uma rede de atores sociais, integrando interesses econômicos, políticos, sociais e ambientais, que o conceito de desenvolvimento sustentável desenvolveu-se, e começou a influenciar o comportamento dos consumidores (LOREK; SPANGENBER, 2014).

Segundo Tachizawa (2009), os consumidores já estão privilegiando, com o comportamento de compra, as empresas que assumem uma postura sustentável. Tal argumento é comprovado em estudos como os de Haws, Winterich e Naylor (2013); Lin e Huang (2012); Hartmann e Apaolaza-Ibáñez (2012); Matthes, Wonneberger e Schmuck, (2014); Koening-Lewis et al (2014); Hoppe et al (2012).

As organizações, cientes dessa nova realidade, passam a adotar "medidas sustentáveis", tais como: estratégias de marketing verde (CHAN, 2013; KO; KIM, 2013; POLONSKY, 2011), readequações na cadeia de suprimentos (TOMASIN et al, 2013; GUPTA; RUDD; LEE, 2014), desenvolvimento de novos produto (GMELIN; SEURING, 2014). E, principalmente, a mensurar o retorno de tais ações como apontam os estudos de Murat (2012), Grimmer e Bingham (2013).

Em suma, a indubitável a importância que tem para as organizações o conhecimento do comportamento do consumidor ecológico (neste estudo os termos ecológico, consciente, sustentável, verde, eco, possuem o mesmo). 
Há uma gama de campos de estudo que buscam compreender os motivos que tornam os consumidores sustentáveis e de que forma essa consciência afeta seus comportamentos de compra. Os focos mais utilizados são: pressões sociais e no status (ELLIOTT, 2013; LEE; ROTMAN; PERKINS, 2014; PIENAAR; LEW; WALLMO, 2013); aspectos demográficos (PUNJ, 2011; POMARICI; VECCHIO, 2014); aspectos funcionais e hedônicos (MATTHES; WONNEBERGER; SCHMUCK, 2014; KOENIGLEWIS et al., 2014) e nos aspectos psicológicos tais como crenças, normas e valores (PUNJ, 2011; PIENAAR; LEW; WALLMO, 2013; HAWS; WINTERICH; NAYLOR, 2013; LIN; HUANG, 2012; HARTMANN; APAOLAZA-IBÁÑEZ, 2012)

Para Laroche et al. (2001) os valores e/ou atitudes são mais importantes para explicar o comportamento ecologicamente orientado do que os aspectos demográficos. Bass e Talarzyk (1972) evidenciaram em seus estudos que a teoria da atitude oferece uma base potencial quando se quer prever o comportamento de compra. A atitude permite prever o comportamento de compra principalmente se os objetos são familiares e se os benefícios que proporcionam são bem conhecidos.

Uma das teorias mais conceituadas sobre valores, atitudes e comportamento é a Teoria do Comportamento Planejado - TCP (Ajzen, 1991) que demonstra que o comportamento humano individual é uma função da intenção de comportamento, sendo que esta depende de outros três quesitos: da combinação das atitudes e a influência que as crenças comportamentais exercem sobre elas, das normas subjetivas e da percepção individual sobre o comportamento percebido.

Em âmbito internacional, a TCP começa a ser utilizada também para a compreensão do consumo ecológico (CHEN; TUNG, 2014; KIM; NJITE; HANCER, 2013; HAN; HSU; SHEU, 2010; HAN; KIM, 2010; LAVAROTA, 2014; TECK, 2013; CHEN et al., 2011). Porém, ainda há grande carência de estudos nacionais que abordam o consumo ecológico por meio da TCP. Dentre os estudos encontrados cita-se o de Hoppe et al. (2012) identificaram o comportamento de consumidores de produtos orgânicos; a pesquisa de Monteiro e Veiga (2006) verificaram a intenção de parar de fumar entre homens e mulheres; o estudo de Veiga, Santos e Lacerda (2006) que buscou identificar a intenção de consumo de cosméticos ecológicos.

Assim, frente à carência de estudos nacionais e com vistas a elucidar os diferentes fatores que impulsionam o consumidor a um comportamento de compra ambientalmente favorável, este estudo tem por objetivo utilizar a Teoria do 
Comportamento Planejado (AJZEN, 1991) para compreender a importância do meio ambiente de processo de compra sustentável. Como objetivos secundários têm-se: verificar a influência das atitudes, normas subjetivas, controle comportamental percebido e intenção de compra no comportamento de compra consciente de professores universitários de cursos de administração.

A escolha por professores universitários deve-se ao fato de que os mesmos são formadores de opinião e que possuem conhecimentos mais profundos sobre Responsabilidade Socioambiental Corporativa e Consumo Ecológico, viso que tais conteúdos estão presentes nos currículos dos cursos de administração.

\section{O CONSUMO E O MEIO AMBIENTE}

Nota-se que há uma tendência em favor do consumo ecologicamente correto, o qual vem sendo cada vez mais praticado pelas pessoas. Na visão de Layrargues (2000), o consumidor verde é alguém que faz suas escolhas de compra levando em consideração não somente qualidade e preço, mas o fato de ser ambientalmente correto, ou seja, para este consumidor o produto não pode ser prejudicial ao ambiente em nenhuma etapa do seu ciclo de vida, pois se acredita que o simples ato da compra determina uma atitude de depredação ou preservação.

De acordo com Akenji (2014), o consumidor ecologicamente correto é aquele que, ao comprar um produto, analisa o impacto que este irá exercer no meio ambiente, preocupando-se com o bem estar social e ambiental e não apenas com sua satisfação. Já para Elliott (2013) o consumidor ambientalmente preocupado procura adotar atitudes e comportamentos de compra coerentes com a conservação dos ecossistemas.

O consumidor consciente não apenas reclama pelos seus direitos, mas também tem deveres a cumprir e atitudes a tomar. Segundo Menon e Menon (1997), surveys relacionadas ao comportamento consumidor nos últimos dez anos revelam um segmento crescente de consumidores que recompensam empresas com reputação positiva com o meio ambiente e que punem empresas que parecem ignorar a questão ambiental. 
O consumo relacionado ao meio ambiente é definido por Dobscha (1993) como uma exibição da responsabilidade no mercado por consumidores que escolhem por produtos e serviços considerados seguros ambientalmente; evitam produtos e serviços não seguros para o meio ambiente ou mal falados; e, apropriadamente, descartam produtos.

Desta forma o consumidor verde procura adotar atitudes e comportamentos de compra coerentes com a conservação dos ecossistemas (ELLIOTT, 2013). Este indivíduo ambientalmente orientado acabará se tornando uma parte permanente da cultura, farão seus valores serem sentidos, acrescentando pressões internas e externas relativas ao "esverdeamento" dos negócios, e acima de tudo, relativas a desempenho ético (OTTMAN, 1994).

A Teoria do Comportamento Planejado (TCP) de Ajzen (1991) incluiu o controle comportamental percebido como um preditor do comportamento, baseando-se na afirmativa de que mantendo a intenção constante, um maior controle percebido irá aumentar a probabilidade de que se desempenhe o comportamento com sucesso (CHEN; TUNG, 2014). Tendo por base que o controle percebido reflete o controle real, este irá influenciar diretamente o comportamento.

A crença da TCP é de que o comportamento humano é guiado por três tipos de crenças: crenças sobre as consequências prováveis de um comportamento (crenças comportamentais), crenças sobre as expectativas normativas de terceiros (crenças normativas) e crenças a respeito da presença de fatores que podem impedir ou facilitar a performance de um comportamento (crenças de controle). Hoyer e MacInnis (2010) colocam que os valores e crenças dos consumidores precisam ser considerados na análise das influências que afetam as decisões de compra.

Dando sequência, as crenças comportamentais produzem uma atitude favorável ou desfavorável em relação ao comportamento, as crenças normativas resultam em pressão social perceptível ou norma subjetiva e crenças de controle podem facilitar ou impedir a performance de um comportamento. Em combinação, atitude, norma subjetiva e percepção de comportamento conduzem à formação de uma intenção comportamental. Quanto mais favoráveis são a atitude e a norma subjetiva e maior o controle percebido, maior deve ser a intenção pessoal de realizar o comportamento. Por fim, dado um suficiente grau de controle do comportamento, 
as pessoas tendem a realizar suas intenções quando as oportunidades aparecem. Por isso, a intenção comportamental é considerada o antecessor imediato do comportamento.

Kalafatis et al (1999) examinaram os determinantes que influenciam as intenções dos consumidores de comprar produtos ambientalmente corretos e concluíram que se pode dizer que a Teoria do Comportamento Planejado de Ajzen (1991) representa um modelo preditivo de intenção de compra.

Com o intuito de compreender o comportamento de consumidores em relação aos produtos pró-ambientais, este estudo aborda o modelo da Teoria do Comportamento Planejado (AJZEN, 2008) e a partir de quatro hipóteses (Figura 1) busca comprovar que as dimensões: atitude, normas subjetivas e controle percebido influenciam a intenção de compra de produtos pró-ambientais, e esta, por sua vez, afeta o comportamento de compra de produtos pró-ambientais. Na sequência, é apresentado o referencial teórico que serviu de base para a formulação das hipóteses.

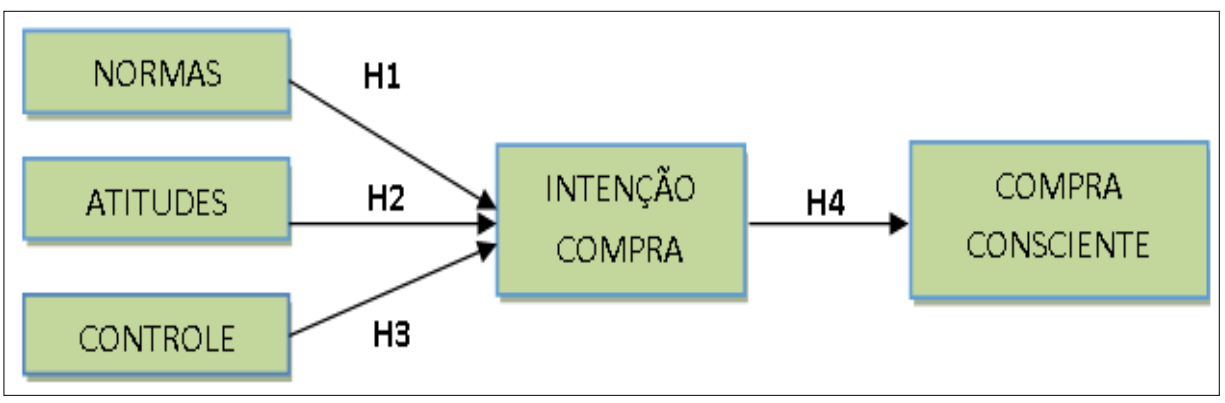

Figura 1. Hipóteses da pesquisa

$\mathrm{Na}$ concepção de Mowen e Minor (2003), as intenções são julgamentos subjetivos sobre como deverá ser o comportamento no futuro em relação à aquisição, disposição e uso de produtos e serviços. As intenções de consumo representam a predisposição de engajar-se em determinada atividade relacionada ao consumo (BLACKWELL; MINIARD; ENGEL, 2008).

As normas subjetivas referem-se às pressões exercidas sobre uma pessoa, visando que a mesma desenvolva determinado comportamento, assim como a sua motivação para atingir tal desempenho (FISHBEIN; AJZEN, 1975). Veiga, Santos e Lacerda (2006) verificaram que a norma subjetiva é fator relevante para explicar a 
intenção de consumir cosméticos ecológicos. Em contraposição, Hoppe et al. (2012) evidenciaram que a associação entre o constructo norma subjetiva e intenção de compra de produtos orgânicos não é significativa.

A partir do exposto, a primeira hipótese busca explorar a relação entre normas subjetivas e intenção de compra de produtos pró-ambientais: H1 - As normas subjetivas impactam positivamente na intenção de compra de produtos pró-ambientais.

Com efeito, Hini, Gendall e Keams (1995) mostram, através de pesquisa quantitativa, que há relacionamento entre atitude e comportamento de indivíduos em relação ao meio ambiente, no entanto, este relacionamento é frágil. A atitude refere-se à predisposição para reagir a determinada situação com uma resposta específica, sendo uma reação aprendida resultante de observações anteriores, de experiências diretas ou da exposição às atitudes dos outros (GILL; CROSBY; TAYLOR, 1986).

Nesse sentido, a segunda hipótese busca verificar o impacto das atitudes em relação a intenção de compra de produtos pró-ambientais: H2 - As atitudes impactam positivamente na intenção de compra de produtos pró-ambientais.

$\mathrm{Na}$ Teoria do Comportamento Planejado (TCP), a Teoria da Ação Fundamentada é estendida para um terceiro fator: controle comportamental percebido (AJZEN, 1991). O controle comportamental percebido se refere à medida em que uma pessoa pensa que suas ações terão um impacto sobre a situação como um todo. A TCP vem sendo bem sucedida quando aplicada no contexto de previsão do comportamento pró-ambiental (CHEUNG; CHAN; WONG, 1999; OREG; KATZ- GERRO, 2006) e implica que o comportamento de compra pró-ambiental do consumidor não seja apenas influenciado pela consciência de uma pessoa, atitudes e pressão social, mas também pela visão individual sobre oportunidades pessoais, que este pode obter, por contribuir com uma determinada questão ecológica (BECHLARSEN, 1996; ÖLANDER; THOGERSEN, 1995).

Hoppe et al. (2012) verificaram que o controle percebido afeta a intenção de compra de produtos orgânicos. De forma similar, Veiga, Santos e Lacerda (2006) constataram que pessoas que têm, supostamente, maior envolvimento com questões ambientais aparentam ter níveis mais elevados de percepção de controle relativo à intenção de uso de cosméticos ecológicos. 
Partindo de tais considerações, a terceira hipótese compreende a relação entre controle percebido e intenção de compra: H3 - O controle percebido impacta positivamente na intenção de compra de produtos pró-ambientais.

De acordo com Ajzen e Fishbein (1980), para compreender profundamente os fatores que afetam o comportamento, é preciso considerar os determinantes dos componentes normativos e de atitude, que, por sua vez, são as crenças individuais que os consumidores têm sobre si próprios e o mundo em que vivem. Consequentemente, as crenças são vistas como determinantes das atitudes e normas subjetivas do indivíduo e, normalmente, determinam as intenções e o comportamento de compra. Nesse sentido, a quarta hipótese se refere a relação entre intenção de compra e comportamento de compra consciente: H4 - A intenção de compra impacta positivamente no comportamento de compra de produtos próambientais.

\section{MÉTODO DO ESTUDO}

O estudo é quantitativo e descritivo, utilizando modelos de mensuração já utilizados e testados anteriormente. A pesquisa foi baseada na escala de Comportamento de Compra Consciente, proposta por Enoki et al. (2008), e na escala de Teoria do Comportamento Planejado, proposta por Veiga et al. (2006).

A coleta de dados utilizou o método online (via Internet) já que a maioria dos respondentes entrevistados reside em outras cidades e outros estados brasileiros. De acordo com Hair et al. (2010), a capacidade dos levantamentos via Internet de alcançarem amostras difíceis de localizar é um fator importante por trás do crescimento deste método. Para a realização da pesquisa foi utilizada a ferramenta forms (formulários) do Google Docs, uma ferramenta de criação de levantamentos online oferecida pela empresa Google Inc. A pesquisa foi criada no Google Docs e posteriormente foi gerado um link (direcionamento) para a pesquisa, o qual foi enviado via e-mail para a amostra selecionada. Junto ao link foi enviado um breve texto com uma explicação do que consiste a pesquisa. Ao final da coleta foram obtidos 454 questionários válidos (sem dados faltantes), respondidos por professores universitários que atuam em cursos de administração, de diversos estados do Brasil.

$\mathrm{O}$ instrumento de coleta de dados foi dividido em três partes: questões 
demográficas; 11 questões sobre o comportamento de compra consciente; 7 questões sobre normas subjetivas, atitudes, controle comportamental e intenção de compra. Utilizou-se uma escala Likert de 5 pontos, sendo que 1 significou a total discordância dos respondentes em relação à assertiva e 05 a total concordância. $\mathrm{O}$ Quadro 1 apresenta os modelos utilizados na pesquisa.

Quadro 1. Modelos utilizados na pesquisa

\begin{tabular}{|c|c|}
\hline Construtos & Assertivas \\
\hline $\begin{array}{l}\text { Comportamento } \\
\text { de compra } \\
\text { consciente }\end{array}$ & $\begin{array}{l}\text { Estou disposto(a) a gastar mais tempo na procura de produtos verdes. } \\
\text { As atuais informações sobre a degradação do meio ambiente despertou a } \\
\text { necessidade e comprar produtos verdes. } \\
\text { Faz parte do meu estilo de vida comprar produtos verdes. } \\
\text { Compro produtos verdes porque tenho consciência que é necessário } \\
\text { preservar o meio ambiente. } \\
\text { Procuro consumir somente produtos que causam menor dano ao meio } \\
\text { ambiente. } \\
\text { Meu comportamento de compra pode ser alterado ao saber que alguns } \\
\text { produtos degradam o meio ambiente. } \\
\text { Minha família influencia para a decisão da aquisiçãa de um produto verde. } \\
\text { A cultura brasileira estimula a compra de produtos verdes. } \\
\text { Compro determinadas marcas verdes para aceitação da sociedade. } \\
\text { Considero primordial na formação do comportamento de compra a } \\
\text { experiência anterior com o produto. } \\
\text { Tenho atitudes positivas em relação aos produtos verdes. }\end{array}$ \\
\hline Atitudes & $\begin{array}{l}\text { Utilizar produtos ecologicamente corretos seria bom } \\
\text { O consumo de produtos ecologicamente corretos traria benefícios ao meio } \\
\text { ambiente. }\end{array}$ \\
\hline $\begin{array}{l}\text { Normas } \\
\text { Subjetivas }\end{array}$ & $\begin{array}{l}\text { As pessoas importantes para mim me aprovariam se eu comprasse apenas } \\
\text { produtos ecologicamente corretos. } \\
\text { Pessoas que são importantes para mim recomendam que eu use produtos } \\
\text { ecologicamente corretos. }\end{array}$ \\
\hline $\begin{array}{l}\text { Controle } \\
\text { Comportamental }\end{array}$ & $\begin{array}{l}\text { Para mim, priorizar a compra de produtos ecologicamente corretos é difícil. } \\
\text { Se eu quiser, posso utilizar apenas produtos ecologicamente corretos }\end{array}$ \\
\hline $\begin{array}{l}\text { Intenção de } \\
\text { Compra }\end{array}$ & $\begin{array}{l}\text { Eu tenho a intenção de utilizar produtos ecologicamente corretos de agora } \\
\text { em diante. }\end{array}$ \\
\hline
\end{tabular}

Fonte: adaptado de Enoki et al. (2008) e Veiga et al. (2006)

Os procedimentos estatísticos adotados seguiram a seguinte sequência: análise de frequência e percentuais para caracterizar os respondentes da pesquisa; 
análise fatorial exploratória para a construção do modelo estrutural; correlações de Pearson para comprovação da validade discriminante do modelo; análise fatorial confirmatória para validação do modelo global; modelagem de equações estruturais para comprovação das hipóteses; teste T para identificação de diferença das médias entre os gêneros; modelagem de equações estruturais para múltiplos grupos, visando identificar as relações causais para o modelo feminino e para o modelo masculino; teste de invariância para identificação das diferenças estatisticamente significativas entre as regressões dos modelos feminino e masculino.

\section{RESULTADOS}

Entre os 454 professores universitários que participaram desta pesquisa, 191 (42,1\%) lecionam em instituições públicas (federal ou estadual) e 262 (57,7\%) em instituições privadas. Em relação ao gênero, variável demográfica mais importante para este estudo, dos respondentes, dos 454 professores, 179 são do sexo feminino $(39,4 \%)$ e 275 (60,6\%) são do sexo masculino.

Quanto à faixa etária, a maioria, 149 respondentes (32,8\%), está na faixa entre 40 e 49 anos, 133 (29,3\%) têm entre 30 e 39 anos, 104 professores (22,9\%) encontra-se na faixa entre 50 e 59 anos, 48 respondentes (10,6\%) têm mais de 59 anos e 20 entrevistados (4,4\%) têm idade inferior à 30 anos. A grande maioria dos respondentes, 330, possui renda mensal familiar é superior a onze salários mínimos.

Sobre o estado civil, 302 respondentes (66,5\%) indicou que é casado(a), 81 professores $(17,8 \%)$ indicaram que são solteiros(as), 49 (10,8\%) são divorciados(as), 14 respondentes $(3,1 \%)$ classificaram-se em outro (estado civil) e 8 respondentes (1,8\%) são viúvos(as). Por fim, a maioria, 297 dos professores (65.4\%), tem filho(s), e 157 (34,6\%) dos respondentes não têm filho(s).

Seguindo os procedimentos sugeridos por Hair et al. (2010), para uma préavaliação do modelo, foi realizada a Análise Fatorial Exploratória (Tabela 1) com rotação varimax e exclusão de itens com cargas e comunalidades inferiores a 0,50.

No modelo de Teoria do Comportamento Planejado, cada um dos constructos (atitude, norma subjetiva e controle do comportamento) foi analisado individualmente. $\mathrm{O}$ constructo atitude permaneceu com duas variáveis (a1 e a2), ambas com cargas de 0,874 , explicando $76,42 \%$ da variância total. $O$ fator apresenta confiabilidade satisfatória, visto que o alpha de Cronbach foi de 0,688 (desejam-se 
valores acima de 0,60). A análise fatorial exploratória mostrou-se adequada, uma vez que o teste de esfericidade de Bartlett foi significativo (valor de 147,818 ) e o valor obtido pelo KMO foi de 0,500 .

O segundo fator do modelo é normas subjetivas, formado por duas variáveis (n1 e n2) que explicam 76,90\% da variância total, apresentam cargas de 0,877 e confiabilidade satisfatória, sendo que o alpha de Cronbach obteve o valor de 0,699. $\mathrm{O}$ KMO alcançou o valor de 0,500 e o teste de esfericidade de Bartlett foi significativo (valor de 154,218).

Tabela 1. Resultados da Análise Fatorial Exploratória

\begin{tabular}{|c|c|c|c|}
\hline \multicolumn{2}{|c|}{ Item } & Carga & Com \\
\hline \multicolumn{4}{|c|}{ Atitudes - Alpha de Cronbach $=0,688$; variância explicada $=76,42 \%$} \\
\hline a1 & Utilizar produtos ecologicamente corretos seria bom & 0,874 & 0,764 \\
\hline $\mathrm{a} 2$ & $\begin{array}{l}\text { O consumo de produtos ecologicamente corretos traria benefícios ao } \\
\text { meio ambiente }\end{array}$ & 0,874 & 0,764 \\
\hline \multicolumn{4}{|c|}{ Normas Subjetivas - Alpha de Cronbach $=0,699 ;$ variância explicada $=76,90 \%$} \\
\hline $\mathrm{n} 1$ & $\begin{array}{l}\text { As pessoas importantes para mim me aprovariam se eu comprasse } \\
\text { apenas produtos ecologicamente corretos }\end{array}$ & 0,877 & 0,769 \\
\hline $\mathrm{n} 2$ & $\begin{array}{l}\text { Pessoas que são importantes para mim recomendam que eu use } \\
\text { produtos ecologicamente corretos }\end{array}$ & 0,877 & 0,769 \\
\hline \multicolumn{4}{|c|}{ Controle Comportamental - Alpha de Cronbach $=0,371$; variância explicada $=61,42 \%$} \\
\hline ct1 & $\begin{array}{l}\text { Para mim, priorizar a compra de produtos ecologicamente corretos é } \\
\text { difícil }\end{array}$ & 0,784 & 0,614 \\
\hline ct2 & Se eu quiser, posso utilizar apenas produtos ecologicamente corretos & 0,784 & 0,614 \\
\hline \multicolumn{4}{|c|}{$\begin{array}{l}\text { Comportamento Compra Consciente } \text { - Alpha de Cronbach }=0,889 ; \text { variância explicada }= \\
64,53 \%\end{array}$} \\
\hline cc1 & $\begin{array}{l}\text { Meu comportamento de compra pode ser alterado ao saber que } \\
\text { alguns produtos degradam o meio ambiente }\end{array}$ & 0,740 & 0,548 \\
\hline $\operatorname{cc} 4$ & $\begin{array}{l}\text { Procuro consumir somente produtos que causam menor dano ao } \\
\text { meio ambiente }\end{array}$ & 0,793 & 0,629 \\
\hline $\operatorname{cc6}$ & Faz parte do meu estilo de vida comprar produtos pró-ambientais & 0,859 & 0,739 \\
\hline $\mathrm{cc} 7$ & $\begin{array}{l}\text { Compro produtos ambientais porque tenho consciência que é } \\
\text { necessário preservar o meio ambiente }\end{array}$ & 0,874 & 0,764 \\
\hline $\operatorname{cc} 8$ & Tenho atitudes positivas em relação aos produtos pró-ambientais & 0,731 & 0,534 \\
\hline cc9 & $\begin{array}{l}\text { Estou disposto(a) a gastar mais tempo na procura de produtos pró- } \\
\text { ambientais }\end{array}$ & 0,812 & 0,659 \\
\hline
\end{tabular}


Controle comportamental é terceiro fator, composto por duas variáveis (ct1 e ct2) que apresentam cargas de 0,784 e explicam $61,42 \%$ da variância total. A confiabilidade não alcançou o valor desejável (acima de 0,60), uma vez que obteve o valor de 0,371 . Embora a análise fatorial exploratória ser satisfatória, sendo que o KMO alcançou o valor de 0,500 e o teste de esfericidade de Bartlett foi significativo (valor de 24,186), optou-se pela exclusão desse constructo do modelo.

No modelo de Comportamento de Compra Consciente, foram excluídas três variáveis por apresentarem cargas inferiores a 0,50: cc3 (A cultura brasileira estimula a compra de produtos pró-ambientais) com carga fatorial de 0,188 ; cc5 (Considero primordial na formação do comportamento de compra a experiência anterior com o produto), com carga de 0,150 e cc11 (Compro determinadas marcas pró-ambientais para aceitação da sociedade), com carga de 0,373 . E duas variáveis foram excluídas por possuírem comunalidades inferiores a 0,50. A variável cc2 (As atuais informações sobre a degradação do meio ambiente despertou a necessidade de comprar produtos pró-ambientais) ficou com comunalidade de 0,468 e a variável cc10 (Minha família influencia para a decisão da aquisição de um produto próambiental) com comunalidade de 0,286.

Dessa forma, o constructo Comportamento de Compra Consciente apresentou uma estrutura unifatorial formada por seis itens (cc1, cc4, cc6, cc7, cc8 e cc9) que explicam 56,32\% da variância total extraída. A análise fatorial exploratória foi satisfatória, uma vez que o teste de esfericidade de Bartlett foi significativo (valor de 1743,641 ) e o KMO de 0,910 . As cargas fatoriais padronizadas variaram de 0,535 (cc10) a 0,864 (cc7) e a confiabilidade do modelo foi muito boa, com alpha de Cronbach de 0,881. Estes resultados encontram-se na Tabela 1.

A ausência de casos extremos foi analisada pelo diagrama em caixa, pois nenhum dos valores discrepantes situou-se a 1,5 ou mais desvios quartílicos distância de mabalanobis - do quartil superior ou inferior. O teste KS e os gráficos Q-Q e P-P demonstraram que alguns itens não apresentavam distribuição normal, o que pode afetar o ajuste do modelo.

$\mathrm{Na}$ Análise Fatorial Confirmatória foi utilizado o método da máxima verossimilhança para a estimação dos parâmetros. O modelo estrutural final (Figura 3 ) apresentou como ínicides de ajuste: chi-quadrado $=212,386$; graus de liberdade 
$=40 ; \chi^{2} / \mathrm{dl}=5,03 ; \mathrm{GFI}=0,922 ; \mathrm{NFI}=0,917 ; \mathrm{RFI}=0,886 ; \mathrm{IFI}=0,932 ; \mathrm{TLI}=$ 0,906; CFI $=0,932 ; \mathrm{RMR}=0,072$ e RMSEA $=0,098$. Segundo Hair et al. (2010), os valores adequados para ajuste do modelo é de RMSEA e RMR inferiores a $0,10, \chi^{2} / \mathrm{dl}$ até 5,00 e valores superiores a 0,900 para os demais indices. Desta forma, o modelo apresentou valores satisfatórios que comprovaram o ajuste do modelo estrutural final.

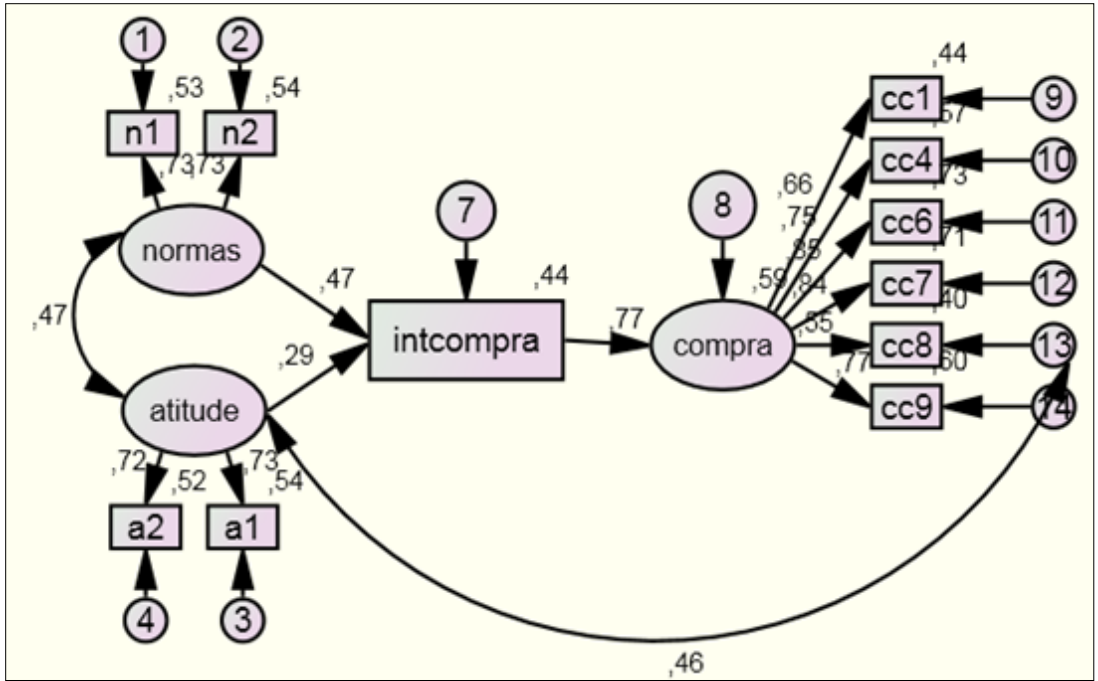

Figura 3. Resultado da Modelagem de Equações Estruturais Fonte: elaborado pelos autores

Para a obtenção dos índices, anteriormente apresentados, foi necessária uma única alteração no modelo estrutural inicial. Seguindo sugestão dos procedimentos de modificação para melhoria dos índices de ajuste, sugeridos pelo próprio software estatístico, foi incluída uma correlação entre a variável cc8 e o constructo atitude (a correlação foi significativa, com valor de 0,463 ). A variável refere-se à assertiva "Tenho atitudes positivas em relação aos produtos pró-ambientais", demonstrando claramente que é uma variável que mensura também atitudes.

A Tabela 2 apresenta os resultados da modelagem de equações estruturais. A comprovação da validade convergente foi constatada, pois todos os itens apresentaram cargas fatoriais padronizadas significativas ao nível de 0,01 . Nenhum item apresentou t-valor abaixo do limite (t-valor $\geq 2,33$ ), sendo que o menor valor 
foi de 10,301 para os itens n1 e n2. A unidimensionalidade do modelo é analisada através dos valores dos resíduos padronizados (erro), e como se observa na tabela, todos os valores são inferiores a 2,58 $(p<0,05)$, sendo o maior valor de 0,110 para a variável a1, o que comprova a unidimensionalidade do modelo.

Tabela 2. Resultado da modelagem de equações estruturais

\begin{tabular}{lll|ccccc}
\hline & Relação & Carga & Carga pad. & Erro & t-valor & Sig. \\
\hline intcompra & $<---$ & normas & 0,561 & 0,472 & 0,077 & 7,265 & $* * *$ \\
intcompra & $<---$ & atitude & 0,529 & 0,295 & 0,105 & 5,026 & $* * *$ \\
Compra & $<---$ & intcompra & 0,441 & 0,768 & 0,031 & 14,276 & $* * *$ \\
a1 & $<---$ & atitude & 1,154 & 0,735 & 0,110 & 10,535 & $* * *$ \\
a2 & $<---$ & atitude & 0,867 & 0,721 & 0,082 & 10,535 & $* * *$ \\
n1 & $<---$ & normas & 1,018 & 0,730 & 0,099 & 10,301 & $* * *$ \\
n2 & $<---$ & normas & 0,983 & 0,733 & 0,095 & 10,301 & $* * *$ \\
cc1 & $<---$ & compra & 0,734 & 0,663 & 0,052 & 14,059 & $* * *$ \\
cc4 & $<---$ & compra & 1,362 & 0,752 & 0,097 & 14,059 & $* * *$ \\
cc6 & $<---$ & compra & 1,665 & 0,853 & 0,108 & 15,378 & $* * *$ \\
cc7 & $<---$ & compra & 1,489 & 0,845 & 0,096 & 15,579 & $* * *$ \\
cc8 & $<---$ & compra & 0,818 & 0,554 & 0,074 & 11,006 & $* * *$ \\
cc9 & $<---$ & compra & 1,540 & 0,772 & 0,107 & 14,400 & $* * *$ \\
\hline
\end{tabular}

**** significativo ao nível de $1 \%$

Visando comprovar a validade discriminante do modelo, foram calculadas as correlações de Pearson entre os constructos (Tabela 3) e o primeiro procedimento foi o de transformar as diversas variáveis que formavam cada fator em uma única variável (cálculo das médias das variáveis). Os dados demonstram que todas as correlações foram significativas ao nível de $1 \%$. Para que a validade discriminante seja comprovada, segundo Hair et al. (2010), as correlações devem ser significativas, porém não podem ser superiores a 0,90 , e os quadrados das correlações (parte superior da matriz) devem ser inferior à variância extraída. A maior correlação encontrada foi de 0,737 entre o fator compra consciente e intenção de compra, cumprindo-se o primeiro critério da validade discriminante. 
$\mathrm{Na}$ análise do segundo critério, calcula-se a variância extraída de cada constructo, por meio de cálculo da carga fatorial quadrática média das variáveis que formam o constructo. Observou-se que a maior quadrado das correlações é de 0,514 (entre os constructos intenção de compra e compra consciente) e que tal valor não foi inferior apenas à variância extraída do constructo norma subjetiva, cujo valor foi de 0,486 . Dessa forma, constatou-se que, pelo critério do valor das correlações, a validade discriminante foi totalmente confirmada e, pelo critério de comparação com a variância extraída, a validade discriminante foi parcialmente constatada (identificou-se problema em um dos quatro constructos).

Tabela 3. Estatísticas descritivas e correlações entre os constructos do modelo

\begin{tabular}{|c|c|c|c|c|c|c|}
\hline & Média $(\sigma)$ & $V E$ & Atitude & Norma & Intenção & Compra \\
\hline Atitude & $4,55(0,62)$ & 0,530 & 1 & 0,111 & 0,194 & 0,241 \\
\hline Norma & $3,11(0,98)$ & 0,486 & $0,334^{* *}$ & 1 & 0,258 & 0,275 \\
\hline Intenção & $3,74(0,80)$ & 0,940 & $0,441^{* *}$ & $0,508^{* *}$ & 1 & 0,514 \\
\hline Compra consciente & $3,64(0,99)$ & 0,558 & $0,491^{* *}$ & $0,524^{* *}$ & $0,717^{* *}$ & 1 \\
\hline Compra consciente & $3,64(0,99)$ & 0,558 & $0,491^{* *}$ & $0,524^{* *}$ & $0,717^{* *}$ & 1 \\
\hline
\end{tabular}

** correlações significativas ao nível de 0,01 .

A Tabela 3 apresenta, também, os valores de estatística descritiva. A escala utilizada variava de 1 a 5 e as maiores médias foram para o constructo atitude (média= $4,55)$, intenção de compra (média $=3,74)$, compra consciente (média=3,74) e norma subjetiva (média $=3,11$ ).

Considerando a escala de 1 a 5, o constructo atitude alcançou a maior média $(4,55)$, demonstrando que os respondentes concordam quanto a utilizar produtos ecologicamente corretos é uma boa iniciativa e que o consumo de produtos ecologicamente corretos traria benefícios ao meio ambiente.

O valor do desvio-padrão demonstra que o constructo onde os respondentes apresentaram maior divergência de opinião foi compra consciente $(\sigma=0,99)$ e a menor divergência foi no constructo norma subjetiva $(\sigma=3,11)$.

Com a validação do modelo, partiu-se para a comprovação das hipóteses, através da modelagem de equações estruturais. A Tabela 4 apresenta os resultados 
das três hipóteses do estudo que propuseram relações entre normas subjetivas e intenção de compra (H1), atitude e intenção de compra (H2) e intenção de compra e compra consciente (H4). Destaca-se que a hipótese 3 não pode ser testada visto que o constructo controle comportamental foi retirado do modelo por não apresentar confiabilidade suficiente (alpha de Cronbach superior a 0,60).

Tabela 4. Teste de hipóteses

\begin{tabular}{llllcccc}
\hline Hip. & \multicolumn{1}{c}{ Relação } & & & $\mathbf{R}$ & $\mathbf{R}^{2}$ & Sig. & Conclusão \\
\hline H1 & Intenção compra & $\beta$ & Normas & 0,406 & 0,165 & $* * *$ & Confirmada \\
H2 & Intenção compra & $\beta$ & Atitude & 0,305 & 0,093 & $* * *$ & Confirmada \\
H3 & Intenção compra & $\beta$ & Controle & $\ldots$ & $\ldots$ & $\ldots$ & Não testada \\
H4 & $\begin{array}{l}\text { Compra } \\
\text { consciente }\end{array}$ & $\beta$ & $\begin{array}{l}\text { Intenção } \\
\text { compra }\end{array}$ & 0,737 & 0,543 & $* * *$ & Confirmada \\
\hline
\end{tabular}

**** significativo ao nível de $1 \%$

A primeira hipótese (H1) afirmava que as normas subjetivas impactam positivamente na intenção de compra de produtos pró-ambientais e os resultados confirmam essa associação, sendo que a relação causal entre os constructos foi de $16,5 \%(\beta=0,165 ; p=0,000)$. Assim, nota-se que os consumidores são influenciados na intenção de compra de produtos pró-ambientais pelas pressões exercidas por terceiros que visam determinar comportamento específico. Os achados de Hoppe et al. (2012) não corroboram esse resultado, uma vez que os autores verificaram que as normas subjetivas não afetam a intenção de compra de produtos orgânicos.

$\mathrm{Na}$ segunda hipótese buscou-se verificar se as atitudes impactam positivamente na intenção de compra de produtos pró-ambientais. Os resultados constatam uma relação significativa e positiva de $9,3 \%(\beta=0,093 ; p=0,000)$, ou seja, os indivíduos que apresentam uma atitude favorável em relação aos produtos ecologicamente corretos tendem a possuir intenção de compra de tais produtos. Veiga, Santos e Lacerda (2006) evidenciaram que a atitude tem maior peso para explicar a intenção de consumir cosméticos ecologicamente corretos, do que a norma subjetiva e o controle percebido.

A terceira hipótese pressuponha que o controle percebido impacta positivamente na intenção de compra de produtos pró-ambientais. No entanto, essa 
relação não foi testada em função do constructo controle comportamental ter sido excluído do modelo por apresentar confiabilidade insuficiente, uma vez que o alpha de Cronbach obtido foi de 0,371, valor inferior ao desejável (alpha de 0,60).

Por fim, a quarta hipótese se referia a intenção de compra impactar positivamente no comportamento de compra de produtos pró-ambientais e os resultados demonstram uma relação significativa e forte, num grau de 54,3\% ( $\beta=$ $0,543 ; p=0,000)$. Dessa forma, observa-se que os consumidores que apresentam intenção de adquirir produtos ecologicamente corretos realmente efetivam sua intenção, ao realizar compra consciente. Na Figura 4, estão apresentados os constructos e suas relações.

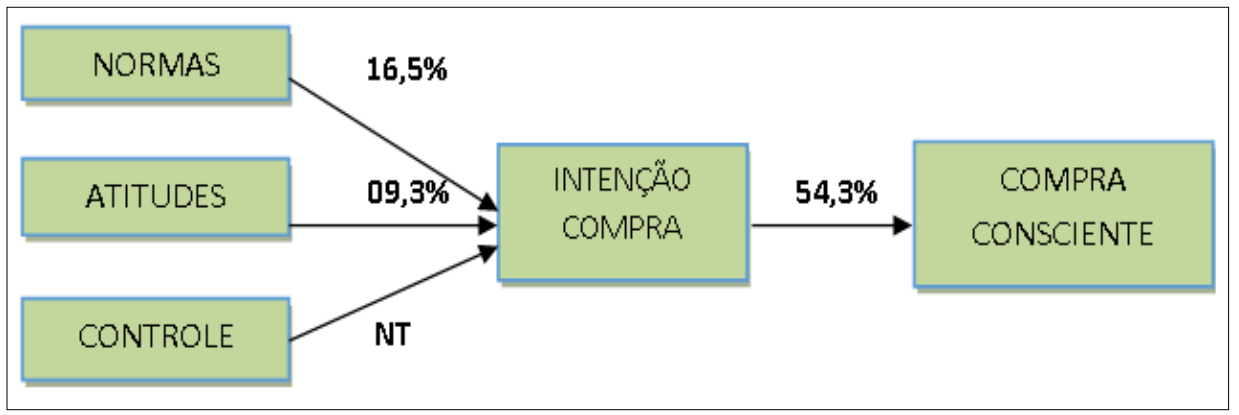

Figura 4. Resumo das hipóteses $\left(\mathrm{R}^{2}\right)$

Na comprovação das hipóteses, verificou-se que três suposições foram confirmadas e uma não foi testada. As hipóteses confirmadas demonstram que as normas subjetivas impactam positivamente na intenção de compra de produtos próambientais; as atitudes influenciam positivamente na intenção de compra de tais produtos; e, a intenção de compra impacta, de forma positiva, na compra consciente.

\section{CONSIDERAÇÕES FINAIS}

O estudo objetivou verificar a influência das atitudes, normas subjetivas, controle comportamental percebido e intenção de compra no comportamento de compra de professores universitários.

Os resultados obtidos comprovaram a validade e a confiabilidade dos 
constructos normas subjetivas, atitudes e intenção comportamental da escala referente à Teoria do Comportamento Planejado, proposta por Veiga et al. (2007); e, do constructo Comportamento de Compra Consciente, modelo proposto por Enoki et al. (2008). Porém, o constructo controle percebido obteve confiabilidade abaixo do mínimo aceitável, não sendo considerado no teste das hipóteses. A robustez do modelo global que atrelou normas subjetivas, atitude, intenção de uso e compra consciente foi comprovada.

A primeira hipótese se referia ao impacto das normas subjetivas na intenção de compra de produtos pró-ambientais e verificou-se que as normas subjetivas são antecedentes da intenção de compra, em um grau de relação de $16,5 \%$. A segunda hipótese demonstrou que as atitudes são antecedentes da intenção de compra de produtos pró-ambientais, num grau de relação de $9,3 \%$.

A terceira hipótese não foi testada, devido o constructo controle percebido ter sido excluído do modelo por apresentar confiabilidade insuficiente. Já a quarta hipótese apresentou a relação mais forte entre as hipóteses, grau de 54,3\%, constando que a intenção de compra impacta positivamente o comportamento de compra de produtos pró-ambientais.

Em suma, conclui-se que os professores pesquisados já desenvolveram preocupações com o meio ambiente e que isto afeta em seus comportamentos de compra.

O estudo apresenta limitações quanto à amostra ser homogênea, uma vez que a pesquisa foi realizada com professores universitários. Neste sentido, sugere-se ampliação de estudos voltados para a questão de compra consciente, englobando diferentes públicos, e acréscimo de construtos que possam contribuir na compreensão do comportamento de consumidores que optam por produtos ecologicamente corretos.

Considera-se o estudo relevante, visto que propiciou contribuições acerca da aplicação da Teoria do Comportamento Planejado para compreensão dos fatores que influenciam a compra consciente, demonstrando ser um modelo adequado, com considerável poder de explicação. A compra consciente é um tema em evidência nos últimos anos, mostrando ser uma tendência de consumo, uma vez que a sociedade está, cada vez mais, preocupada com os impactos ambientais decorrentes de suas ações. 


\section{REFERÊNCIAS}

AJZEN, I. The theory of planned behavior: some unresolved issues. Organizational Behavior and Human Decision Processes, v. 50, p. 179-211, 1991.

AJZEN, I. Consumer attitudes and behavior. In: C.P. HAUGTVEDT; P.M. HERR; F.R. CARDES (Ed.). Handbook of consumer psychology. New York: Lawrence Erlbaum Associates, 2008. p. 525-548.

AJZEN, I. Perceived behavioral control, self-efficacy, locus of control, and the theory of planned behavior. Journal of Applied Social Psychology, v. 32, p. 665-683, 2002.

AJZEN, I.; FISHBEIN, M. Understanding attitudes and predicting social behavior. Englewood Cliffs: Prentice-Hall, 1980. 278p.

AKENJI, L. Consumer scapegoatism and limits to green consumerism. Journal of Cleaner Production, v.63, n.15, p.13-23, 2014.

BASS, F. M.; TALARZYK, W. W. An attitude model for the study of brand preference, Journal of Marketing Research, v. 9, n. 1, p. 93-96, february, 1972.

BECH-LARSEN, T. Danish consumers' attitudes to the functional and environmental characteristics of food packaging. Journal of Consumer Policy, v. 19, p. 339-363, 1996.

BLACKWELL, R. D.; MINIARD, P. W.; ENGEL, J. F. Comportamento do consumidor. 9. ed. Rio de Janeiro: Cengage Learning, 2008.

CHAN, E. S. W. Managing green marketing: Hong Kong hotel managers' perspective. International Journal of Hospitality Management, v.34, p.442-461, 2013.

CHEUNG, S. F.; CHAN, D. K. S.; WONG, Z. S. Y. Reexamining the theory of planned behaviour in understanding wastepaper recycling. Environment and Behavior, v. 31, p. 587-612, 1999. 
CHEN, C-J; GREGOIR, M.; ARENDT, S.; SHELLEY, M. College and university dining services administrators' intention to adopt sustainable practices: results from US institutions. International Journal of Sustainability in Higher Education, v.12, n.12, p.145-162, 2011.

CHEN, M-F; TUNG, P-J. Developing an extended Theory of Planned Behavior model to predict consumers' intention to visit green hotels. International Journal of Hospitality Management, v.36, p. 221-230, 2014.

DOBSCHA, S. Woman and the environment: applying ecofeminism to environmentally: related consumption. Advances in Consumer Research, v. 20, p 36-40, 1993.

ELLIOTT, R. The taste for green: The possibilities and dynamics of status differentiation through "green" consumption. Poetics, v.41, n.3, p. 294-322, 2013.

ENOKI, P. A.; ADUM, S. H. N.; FERREIRA, M. Z.; VELDEVINO, S. L.; AURELIANO, C. A.; SILVA, A. A. Estratégias de marketing verde na percepção de compra dos consumidores na grande São Paulo. In: ENCONTRO DE MARKETING DA ANPAD, III EMA, 2008. Anais... Curitiba: ANPAD, 2008.

FISHBEIN, M.; AJZEN, I. Belief, Attitude, Intention, and Behavior: An Introduction to Theory and Research. Reading, MA: Addison-Wesley, 1975.

GILL, J. D.; CROSBY, L.; TAYLOR, J. R. Ecological concerns attitudes and social norms in voting behavior. Public Behavior Quarterly, v. 50, n. 4, p. 537-554, 1986.

GMELIN, H.; SEURING, S. Determinants of a sustainable new product development. Journal of Cleaner Production, v.69, p.1-9, 2014.

GRIMMER, M.; BINGHAM, T. Company environmental performance and consumer purchase intentions. Journal of Business Research, v.66, n.10, p.1945-1953, 2013.

GUPTA, S.; RUDD, J.; LEE, N. Business sustainability through successful integration of marketing and operations. Industrial Marketing Management, v.43, n.1, p.3-5, 2014. 
HAN, H.; HSU, L-T; SHEU, C. Application of the Theory of Planned Behavior to green hotel choice: Testing the effect of environmental friendly activities. Tourism Management, v.31, n.3, p.325-334, 2010.

HAN, H.; KIM, Y. An investigation of green hotel customers' decision formation: Developing an extended model of the Theory of Planned Behavior. International Journal of Hospitality Management, v.29, n.4, p.659-668, 2010.

HAIR, JR., J. F.; ANDERSON, R. E.; TATHAM, R. L.; BLACK, W. C. Análise Multivariada de Dados. 5. ed. Porto Alegre, Bookman, 2010.

HARTMANN, P.; APAOLAZA-IBÁÑEZ, V. Consumer attitude and purchase intention toward green energy brands: The roles of psychological benefits and environmental concern. Journal of Business Research, v.65, n.9, p.1254-1263, 2012

HAWS, K.; WINTERICH, K. P.; NAYLOR, R. W. Seeing the world through GREENtinted glasses: Green consumption values and responses to environmentally friendly products. Journal of Consumer Psychology, nov. 2013.

HINI, D.; GENDALL, P.; KEAMS, Z. The link between environmental attitudes and behavior, Marketing Bulletin, v. 6, p. 22-31, 1995.

HOPPE, A.; BARCELLOS, M. D.; VIEIRA, L. M.; MATOS, C. A. Comportamento do Consumidor de Produtos Orgânicos: uma Aplicação da Teoria do Comportamento Planejado. BASE - Revista de Administração e Contabilidade da Unisinos, v. 9, n. 2, p. 174-188, 2012.

HOYER, W.; McINNIS, D. Consumer behavior. Australia South-Western: Cengage Learning, 2010.

KALAFATIS, S. P.; POLLARD, M.; EAST, R.; TSOGAS, M. H. Green marketing and Ajzen's theory of planned behaviour: a cross-market examination. Journal of Consumer Marketing, v. 16, n. 5, p. 441-460, 1999.

KIM, Y. J.; NJITE, D.; HANCER, M. Anticipated emotion in consumers' intentions to select eco-friendly restaurants: Augmenting the Theory of Planned Behavior. International Journal of Hospitality Management, v.34, p.255-262, 2013. 
KO, E.; HWANG, K.; KIM, E. Green marketing' functions in building corporate image in the retail setting. Journal of Business Research, v.66, n.10, p.1709-1715, 2013

KOENIG-LEWIS, V.; PALMER, A.; DERMODY, J; URBYE, A. Consumers' evaluations of ecological packaging - Rational and emotional approaches. Journal of Environmental Psychology, v.37, p.94-105, 2014.

LAROCHE, B; TOMIUK, M.; BERGERON, J.; BARBARO-FERLEO, G. Targeting consumers who are willing to pay more for environmentally friendly products. Journal of Consumer Marketing, n. 6, v. 18, p. 503-20. 2001.

LAVAROTA, L. Influence of retailers' commitment to sustainable development on store image, consumer loyalty and consumer boycotts: Proposal for a model using the Theory of Planned Behavior. Journal of Retailing and Consumer Services, 2014.

LAYRARGUES, P. P. Sistemas de gerenciamento ambiental, tecnologia limpa e consumidor verde: a delicada relação empresa-meio ambiente no ecocapitalismo. Revista de Administração de Empresas, v. 40, n. 2, p. 80-88, 2000.

LEE, M.; ROTMAN, J.; PERKINS, A. Embodied cognition and social consumption: Self-regulating temperature through social products and behaviors. Journal of Consumer Psychology, v.24, n.2, p.234-240, 2014.

LIN, P-C; HUANG, Y.W. The influence factors on choice behavior regarding green products based on the theory of consumption values. Journal of Cleaner Production, v. 22, n.1, p. 11-18, 2012.

LOREK, S.; SPANGENBERG, J. Sustainable consumption within a sustainable economy - beyond green growth and green economies. Journal of Cleaner Production, v.63, n.15, p. 33-44, 2014.

MATTHES, J.; WONNEBERGER, A.; SCHMUCK, D. Consumers' green involvement and the persuasive effects of emotional versus functional ads. Journal of Business Research, v. 67, n. 9, p. 1885-1893, 2014. 
MENON, A.; MENON, A. Enviropreneurial marketing strategy: the emergence of corporate environmentalism as market strategy. Journal of Marketing, v. 61, p. 51-67, 1997.

MONTEIRO, P.; VEIGA, R. Personalidade e consumo: uma avaliação empírica do modelo metateórico de motivação e personalidade. In: ENCONTRO DAASSOCIAÇÃO NACIONAL DOS PROGRAMAS DE PÓS-GRADUAÇÃO EM ADMINISTRAÇÃO, ENANPAD, 30., Salvador. Anais... Salvador: ANPAD, 2006.

MOWEN, J. C.; MINOR, M. S. Comportamento do consumidor. São Paulo: Prentice Hall, 2003.

MURAT, I. A. The impact of green product innovation on firm performance and competitive capability: the moderating role of managerial environmental concern. Procedia - Social and Behavioral Sciences, v. 62, p. 854-864, 2012.

OREG, S.; KATZ-GERRO, T. Predicting proenvironmental behavior cross-nationally: values, the theory of planned behavior and value-belied-norm theory. Environment and Behavior, v. 38, p. 462-483, 2006.

ÖLANDER, F.; THOGERSEN, J. Understanding of consumer behaviour as a prerequisite for environmental protection. Journal of Consumer Policy, v. 18, p. 345-385, 1995.

OTTMAN, J. A. Marketing verde: desafios e oportunidades para a nova era do marketing. São Paulo: Makron Books, 1994.

PIENAAR, E.; LEW, D.; WALLMO, K. Are environmental attitudes influenced by survey context? An investigation of the context dependency of the New Ecological Paradigm (NEP) Scale. Social Science Research, v. 42, n. 6, p. 1542-1554, 2013.

POMARICI, E.; VECCHIO, R. Millennial generation attitudes to sustainable wine: an exploratory study on Italian consumers. Journal of Cleaner Production, v. 66, p. 537-545, 2014. 
PUNJ, G. Effect of consumer beliefs on online purchase behavior: the influence of demographic characteristics and consumption values. Journal of Interactive Marketing, v. 25, n. 3, p. 134-144, 2011.

POLONSKY, M. J. Transformative green marketing: Impediments and opportunities. Journal of Business Research, v. 64, n. 12, p. 1311-1319, 2011.

TACHIZAWA, T. Gestão ambiental e responsabilidade social corporativa: estratégias de negócios focadas na realidade brasileira. 6. ed. São Paulo: Atlas, 2009.

TECK, T. Use of Structural Equation Modeling to Predict the Intention to Purchase Green and Sustainable Homes in Malaysia. Asian Social Science, v. 9, n. 10, p. 1911-2017, 2013.

TOMASIN, L.; PEREIRA, G. M.; BORCHARDT, M.; SELLITTO, M. A. How can the sales of green products in the Brazilian supply chain be increased? Journal of Cleaner Production, v. 47, p. 274-282, 2013.

TSARENKO, Y.; FERRARO, C.; SANDS, S.; MCLEOD, C.Environmentally conscious consumption: The role of retailers and peers as external influences. Journal of Retailing and Consumer Services, v. 20, n. 3, p. 302-310, 2013

VEIGA, R. T.; SANTOS, D. O.; LACERDA, T. S. Antecedentes da Intenção de Consumo de Cosméticos Ecológicos. In: ENCONTRO DE MARKETING DA ANPAD, 2 EMA, 2006, Rio de Janeiro. Anais... Rio de Janeiro: ANPAD, 2006.

Recebido em: 03 de agosto de 2014 Aceito em: 21 de janeiro de 2016 